\title{
SONG, DANCE, AND SMELL ROUTINE? INTERPRETING THE CONTENT AND FUNCTION OF MULTIMODAL SIGNALS IN A SONGBIRD, THE DARK-EYED JUNCO (JUNCO HYEMALIS)
}

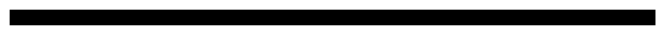

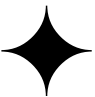 \\ DUSTIN G. REICHARD $\uparrow$ MARINE DROUILLY $\uparrow$ INDIANA UNIVERSITY $\uparrow$ BLOOMINGTON \\ DANIELLE J. WHITTAKER $\uparrow$ MICHIGAN STATE UNIVERSITY $\uparrow$ EAST LANSING \\ STEPHEN FERGUSON $\downarrow$ COLLEGE OF WOOSTER $\uparrow$ WOOSTER
}

\begin{abstract}
$\uparrow \quad$ ABSTRACT
The study of animal communication has been dominated by a focus on signal types that are easily recognized and quantified by human observers. This approach has inevitably limited our ability to identify cryptic signals such as low-amplitude vocalizations and signals that transmit beyond the range of our sensory system, such as most olfactory signals. Only recently with the development of new technologies and less biased sampling techniques have we begun to unravel the importance and function of these non-traditional signal types. Here we report the results of two experiments focusing on poorly studied signals using a common songbird, the dark-eyed junco. We investigated the effect of lowamplitude song on male physiology and the occurrence of bill-wiping behavior during courtship and aggressive interactions. Preliminary results suggest that males do not alter their plasma testosterone or corticosterone levels in response to a song playback of high-amplitude or low-amplitude song, indicating that a stronger stimulus may be necessary to affect circulating hormones. Males that received intrusions of a live male or female conspecific performed significantly more bill-wiping in response to the female conspecific, suggesting that bill-wiping may be an important and overlooked courtship signal in this species.
\end{abstract}

\section{$\uparrow \quad$ INTRODUCTION}

Animal communication is dependent on a variety of acoustic, visual, and olfactory signals for the successful transmission of information, such as the signaler's behavioral intent or potentially the inherent qualities of the signaler (McGregor 2005, Searcy and Nowicki 2005). Owing to the abundance of signal types available, receivers of both sexes are often presented with multiple signals of varying types or "multimodal signals" that must be interpreted in order to gain the maximum amount of information about the signaler. These signals may be redundant and all contain the same or similar information, or they may be unique and each relate to different aspects of the signaler. For example, one signal may function primarily to identify the species of the sender, while another may transmit information about that individual's quality (Hebets and Papaj 2005). Alternatively, one signal alone may be used deceptively (e.g., suggesting the sender is of a high quality) while the integration of multiple signals may give the receiver more accurate information (Ward and Mehner 2010). Understanding the function and information content of all potential signal types is critical for deciphering the full content of a multimodal display, such as courtship behavior.

Male Dark-eyed Juncos (Junco hyemalis), a common North American songbird, produce two distinct classes of song: (1) high amplitude (loud), long-range song (LRS) that functions in mate 
attraction and territoriality, and 2) low-amplitude (soft), short-range song (SRS) that is substantially more complex than LRS and functions predominantly in courtship (Titus 1998, Reichard et al., 2011). Previous work has shown that male juncos respond significantly more aggressively to a territorial intrusion containing SRS than to an intrusion of LRS or soft LRS (Reichard et al., 2011). The striking difference in aggression towards SRS raises the question of whether males also experience a distinct physiological response to SRS. Males of many species, including juncos (McGlothlin et al., 2007), are known to elevate their circulating testosterone (T) in response to a territorial challenge containing LRS, while males of other species experience a similar surge in corticosterone (a hormone typically associated with the stress response) rather than $\mathrm{T}$ (reviewed in Goymann 2009). To investigate the hormonal response of male juncos to each song class, we performed territorial intrusions with LRS or SRS, quantified aggressive behavior during the intrusion, and captured males post-intrusion to take a blood sample for later hormone analyses. Additionally, to better understand the importance and function of LRS and SRS we attached microphone-transmitters (see below) to free-living male juncos to allow us to observe their natural singing behavior under unmanipulated conditions.

Another objective of this study was to investigate behavior related to chemical communication in juncos. Birds perform a "billwiping" behavior during courtship and aggressive interactions, in which they wipe their bills on the perch/branch/other substrate. This same motion is used to clean the bill after eating, but is considered "irrelevant" or displacement behavior in social contexts (Clark, 1970). We have observed in the lab that this motion seems to leave preen oil on perches, and we hypothesize that this is actually a scentmarking behavior.

Additionally, we have found that in two-way choice tests, female juncos prefer the scent of smaller males over that of larger males, and that odor does show a relationship with body size in male juncos (Whittaker et al., 2011). We predicted that smaller males, who are typically less visually attractive to females (Hill et al., 1999), would invest more in chemical communication during courtship, either by producing larger quantities of preen oil (and thus having a larger preen gland) or behaviorally by increasing the frequency of bill-wiping. Similar effects have been observed in insects (Thomas \& Simmons, 2011) and sticklebacks (Candolin, 2000).
We tested the following hypotheses: 1) bill-wiping is a form of scent-marking important in competitive interactions and courtship and 2) smaller males invest more effort in chemical signaling to attract females.

\section{METHODS}

\section{Song Study}

\section{Hormonal response to song playback}

We conducted song playbacks with 40 male dark-eyed juncos near the AMK Ranch, the Grand View Point Trail, and south of the Death Canyon trailhead in Grand Teton National Park. 20 males received a song playback of low-amplitude, shortrange song (SRS) and 20 males received playback of high-amplitude long-range song (LRS). All playbacks were created using methods previously reported by Reichard et al. (2011). For this study, all song playbacks were $10 \mathrm{~min}$ in duration. We quantified male behavior during playback and for an additional 10 min post-playback.

At the completion of the playback and 10 min post-playback observation period, we unfurled a mist net and revealed a live, male conspecific and attempted to capture the focal male. Immediately following capture we rapidly collected 50-200 ul of blood from the alar vein using a micro-hematocrit tube. Birds were them placed in a bag to simulate restraint-induced stress for $15 \mathrm{~min}$. We then took a second blood sample of approximately $50 \mathrm{ul}$ from the opposite wing to examine stress-induced hormone levels. Birds were then banded, measured, and released.

Control males were caught rapidly (> $5 \mathrm{~min}$ of playback) and bled rapidly using the same protocol as described above to allow for an estimated of baseline hormone levels. Control males did not receive the handling stress treatment and were released immediately after blood sampling and banding.

All blood samples were stored on ice before being centrifuged. Hormone-containing plasma was then drawn off with a Hamilton syringe and frozen at $-20^{\circ} \mathrm{C}$ for transport to Indiana University. We calculated testosterone and corticosterone concentrations in our plasma samples using commercially available Enzyme Immune Assay (EIA) kits from Cayman Chemical. 


\section{Radiotelemetry}

We monitored vocalizations and movements of male dark-eyed juncos using a newly developed type of radiotransmitter, the microphone-transmitter (Sparrow Systems, Inc., Fisher, Illinois). Microphone-transmitters are different from traditional radiotransmitters in that they contain a tiny condenser microphone that records and transmits every vocalization produced by the subject as well as the traditional pulse of tones important in locating the subject. Transmitters were attached by first gluing the transmitter to a small piece of cloth and then gluing the cloth+transmitter to the back of the bird after trimming a small patch of feathers. We recaptured males and removed transmitters after the battery died.

After each mic-transmitter was attached we tracked the individual for a minimum of two hours each day typically divided between early morning (0500-0700) and afternoon (1300-1700). During active tracking we took copious observations of the individual's behavior, location, and social interactions. A stationary antenna continued to record vocalizations independently of our behavioral observations for the entire life of the transmitter.

\section{Olfaction Study}

\section{Preen Gland Size}

We measured the uropygial gland of all captured juncos following Moreno-Rueda (2010): after carefully wiping the feathers away from the gland using an alcohol wipe, we used digital calipers to measure the length, width, and height from the base of the gland to the base of the papilla. We estimated preen gland volume by multiplying the three measurements.

\section{Bill-wiping in response to a male or female intruder}

We conducted playback trials using a very similar method as the song study. Thirty male subjects who were naïve to playback received two trials, at least 45 minutes apart, in random order. One trial consisted of a male lure paired with a playback of male LRS, and the other trial used a female lure paired with playback of a female trill (a sound often given by females who are interested in mating). Playback protocol was as follows:

1. Attraction playback (LRS or female trill) to alert the subjects to our presence - up to 10 minutes, until the subject has reached the playback arena;
2. Five minutes of the treatment playback, during which we recorded the subject's behavior;

3. Five minutes of silence during which we continued to record the subject's behavior.

Trials were video-recorded, and a shotgun microphone was also used to record any vocalizations made by the subjects. At the end of the trials, we captured the subject with a mistnet, banded it (if not already banded) and took morphological measurements. We also took a preen oil sample for later chemical analyses, stored at $-20^{\circ} \mathrm{C}$ until transported to Indiana University, and a small blood sample $(\sim 200 \mu \mathrm{l})$ from the alar vein for later genetic analyses, stored at $4^{\circ} \mathrm{C}$ until transported to IU.

\section{Data analysis}

Videos were scored for the following behaviors: latency to approach lure within 5 meters and within 1 meter, time spent within 5 meters and 1 meter, latency to song, number of LRS, seconds of SRS, and number of bill-wipes, tail-spreads, and flights over the cage. All data were analyzed in SPSS 20 .

\section{Preliminary Results}

\section{Song Study}

\section{Hormonal response to song playback}

Males spent significantly more time within 5 $\mathrm{m}(t$-test; $t(1,38)=-4.26, \mathrm{P}<0.001)$ and $1 \mathrm{~m}(t$ $(1,38)=-5.01, \mathrm{P}<0.001)$ of the speaker when responding to SRS than to LRS (Figure 1). Males performed significantly more flights when responding to $\operatorname{LRS}(t(1,38)=4.00, \mathrm{P}<0.001)$, but did not differ in the number of songs produced in response to each playback type $(t(1,38)=-0.79, \mathrm{P}=$ 0.437).

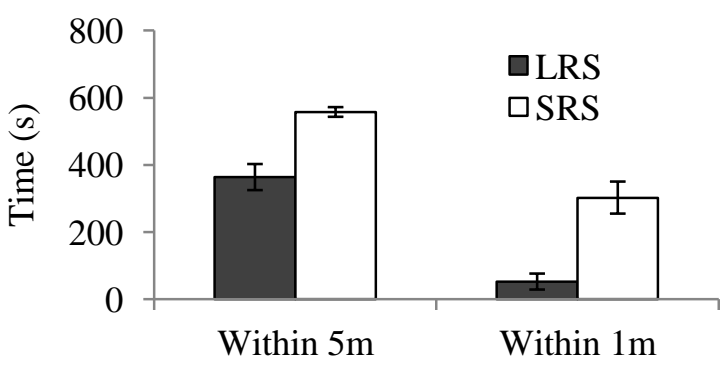

Figure 1. Mean total time spent within $5 \mathrm{~m}$ and $1 \mathrm{~m}$ of the speaker during a 10 min playback of long-range song (LRS) or short-range song (SRS). 
Plasma testosterone did not differ statistically between treatment groups (Figure 2; One-way ANOVA; $\mathrm{F}(2,57)=0.587, \mathrm{P}=0.559)$.

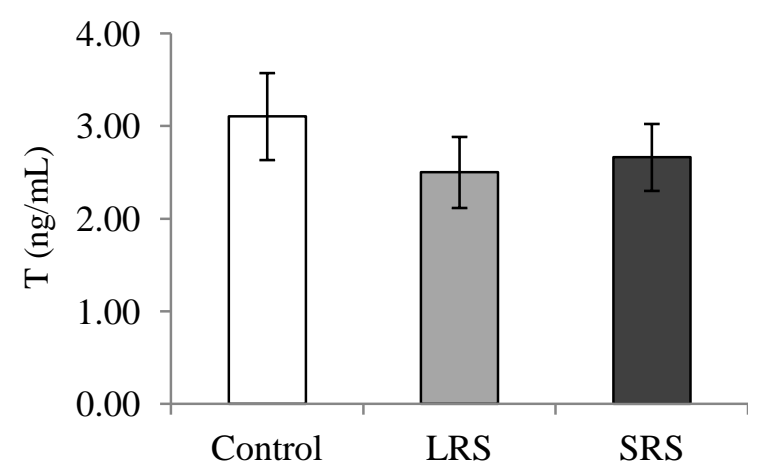

Figure 2. Mean plasma testosterone (T) measured following a rapid capture (control) or 10-min playback with LRS or SRS.

Plasma corticosterone did not differ statistically between treatment groups (Figure 3; One-way ANOVA; $\mathrm{F}(2,55)=1.039, \mathrm{P}=0.360)$. Males had significantly higher levels of corticosterone post-handling stress (Repeated Measures $t$-test; $t(1,35)=-19.75, \mathrm{P}<0.001)$ than post-STI. Males did not differ in the change in corticosterone between the post-STI sample and posthandling stress sample when compared between LRS and SRS treatments $(t(1,34)=-0.249, \mathrm{P}=0.805)$.

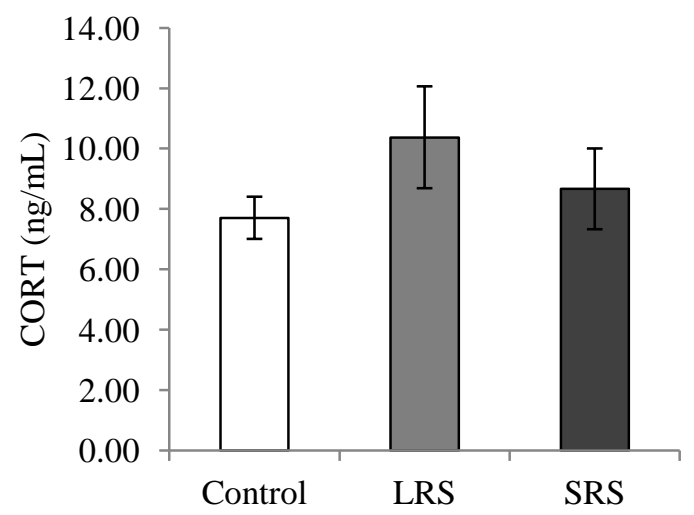

Figure 3. Mean plasma corticosterone (CORT) measured following a rapid capture (control) or 10-min playback with LRS or SRS.

\section{Radiotelemetry}

We attached mic-transmitters to 9 free-living male juncos located at or near the AMK Ranch. Data from the transmitters are still being analyzed, but we were able to record numerous examples of lowamplitude vocalizations from mated and unmated individuals in a variety of social contexts.

\section{Olfaction Study}

We conducted trials at 30 territories. One of these trials was deleted from the analysis because the responding subject was a female, leaving a sample size of 29 males. We also measured preen glands for 18 females and 59 males.

\section{Bill-wiping}

Males bill-wiped significantly more in response to female intruders (per 10 minute trial: mean $6.0 \pm 1.6)$ than in response to male intruders $(1.66 \pm 0.33$, one-way ANOVA, $p=0.015)$. When courting females, males showed a strong positive correlation between frequency of bill-wiping and frequency of tail-spreading, a behavior observed during courtship in which a male spreads his tail feathers to display his tail-white (Figure 4, Pearson correlation $=0.657, p<0.001)$. Males in their first adult year bill-wiped more frequently in response to females (mean bill-wipes, $13.0 \pm 2.98$ ) than older males did $(3.0 \pm 1.06$, one-way ANOVA, $\mathrm{p}=0.03)$. Wing length was negatively correlated with billwiping frequency in response to females (Figure 5, Pearson correlation $=-0.603, \mathrm{p}=0.01)$. No correlations between bill-wiping in response to male intruders and any other behavioral or morphological characteristic was observed. Bill-wiping in response to males was not correlated with bill-wiping in response to females (Figure 6, Pearson correlation $=$ $1.20, \mathrm{p}=0.534)$.

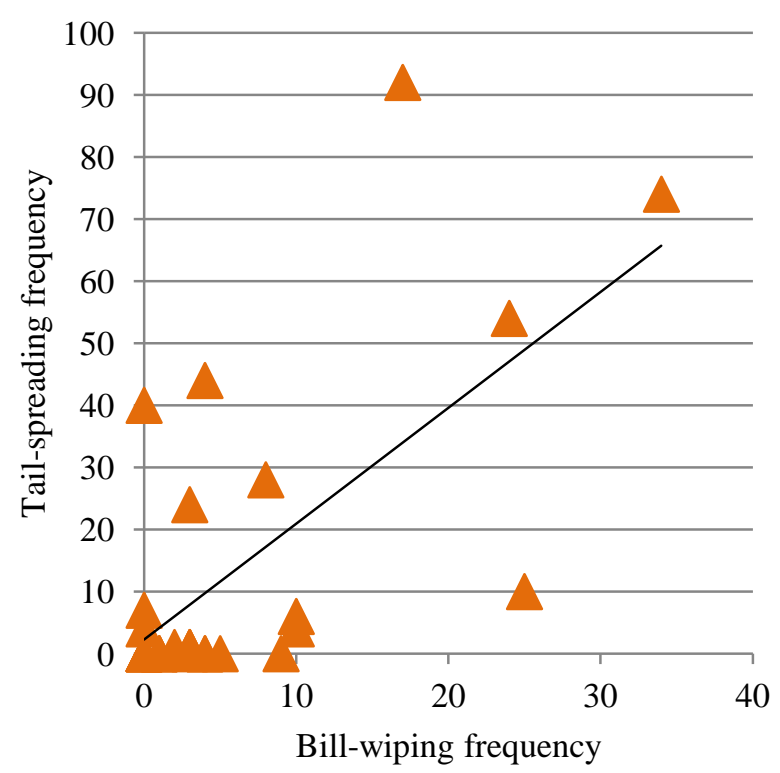

Figure 4. Positive relationship between bill-wiping frequency and tail-spreading frequency. 


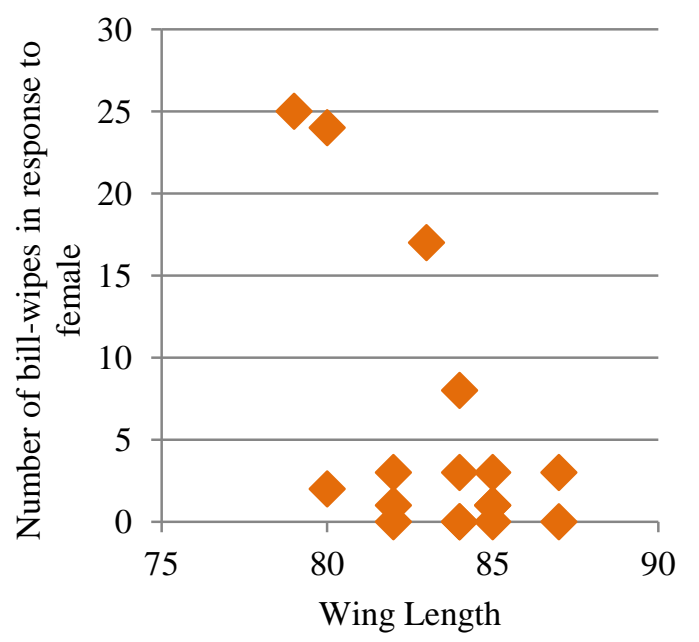

Figure 5. Smaller males tend to bill-wipe more frequently in response to females than larger males.

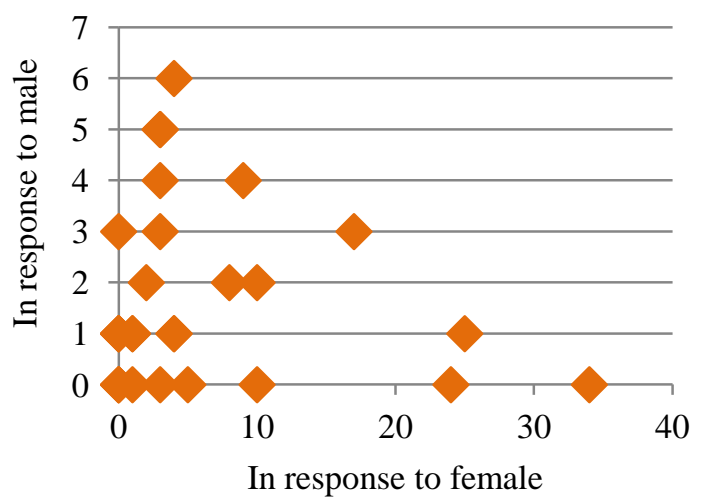

Figure 6. Individual variation in bill-wiping was not consistent across contexts.

\section{Preen gland size}

Female preen gland volume was larger than that of males (females: $171 \pm 46 \mathrm{ml}$; males: $145 \pm 42$ $\mathrm{ml}$; one-way ANOVA, $\mathrm{p}=0.03$ ). In females, preen gland volume increased with Julian date (Pearson correlation $=0.629, p=0.005)$. In males, volume did not correlate with Julian date; the only individual preen gland measurement that correlated with date in males was height (Pearson correlation $=0.351, \mathrm{p}=$ 0.006). Male preen gland size was not correlated any of the behavioral or morphological measurements in this study.

\section{$\uparrow \quad$ DISCUSSION}

Our results highlight the importance of inconspicuous and poorly studied signal classes in mediating social interactions between songbirds and all animal taxa. Although male juncos did not differ in their hormonal response to LRS or SRS, males did respond significantly more strongly to SRS than LRS. Thus, SRS is actually a more potent elicitor of aggressive behavior than the more thoroughly studied LRS, which is the song of choice for the vast majority of studies focusing on aggressive behavior in songbirds. In the case of SRS, males may be responding more aggressively because SRS is signaling a courtship event on their territory, which could result in the territory owner being cuckolded by an intruding male (Reichard et al., 2011). The lack of increase in plasma testosterone or corticosterone is surprising given the robust behavioral responses to the song stimuli. Previous studies investigating the hormonal response to song playback have also included a visual stimulus such as a live conspecific or taxidermy mount, which suggests that simply hearing male song, regardless of the level of threat, does not result in changes in physiology (McGlothlin et al. 2007, Goymann 2009).

Our data also suggest that bill-wiping is an important part of junco courtship behavior. Males performed bill-wiping significantly more often in response to female intruders than to male intruders, and the frequency of bill-wiping increased with courtship intensity. Bill-wiping does not appear to be related to the individual's personality, since there was no correlation between an individual's bill-wiping frequency in the two different contexts. Smaller and younger males did invest more effort into bill-wiping in response to females. However, male preen gland size did not correlate with body size or any other morphological measure; nor did it correlate with any behavioral measure. Thus, if smaller males do invest more in an attractive chemical signal, it is through behavior, not through production of preen oil.

\section{$\uparrow \quad$ ACKNOWLEDGEMENTS}

We thank Elizabeth Schultz, Rebecca Koch, and Eric Snajdr for assistance in the field. This study was funded by grants from the National Science Foundation to Ellen D. Ketterson (IOS-0820055) and Dustin Reichard (IOS-1011145). Stephen Ferguson was supported by the NSF Research Experience for Undergraduates program and Dustin Reichard was supported by a NSF Graduate Research Fellowship. Danielle Whittaker was supported by the BEACON Center for the Study of Evolution in Action, an NSF Science and Technology Center (DBI-0939454). 
$\downarrow \quad$ LITERATURE CITED

Candolin U. 2000. Increased signaling effort when survival prospects decrease: male-male competition ensures honesty. Animal Behaviour. 60:417-422.

Clark GA Jr. 1970. Avian Bill-Wiping. The Wilson Bulletin 82:279-288.

Goymann W. 2009. Social modulation of androgens in male birds. General and Comparative Endocrinology. 163:149-157.

Hebets EA, Papaj DR. 2005. Complex signal function: developing a framework of testable hypotheses. Behavioral Ecology and Sociobiology. 57:197-214.

Hill JA, Enstrom DA, Ketterson ED, Nolan V Jr. Ziegenfus C. 1999. Mate choice based on static vs. dynamic secondary sexual traits in the dark-eyed junco. Behavioral Ecology. 10:91-96.

McGregor PK. Animal Communication Networks. Cambridge: Cambridge University Press; 2005.

McGlothlin JW, Jawor JM, Ketterson ED. 2007. Natural variation in a testosterone-mediated trade-off between mating effort and parental effort. American Naturalist. 170:864-875.

Moreno-Rueda G. 2010. Uropygial gland size correlates with feather holes, body condition and wingbar size in the house sparrow Passer domesticus. Journal of Avian Biology. 41:229-236.
Reichard DG, Rice RJ, Vanderbilt CC, Ketterson ED. 2011. Deciphering information encoded in birdsong: male songbirds with fertile mates respond most strongly to complex, lowamplitude songs used in courtship. American Naturalist. 178:478-487.

Searcy W, Nowicki S. The evolution of animal communication: reliability and deception in signaling systems. Princeton and Oxford: Princeton University Press; 2005.

Thomas ML, Simmons LW. 2011. Short-term phenotypic plasticity in long-chain cuticular hydrocarbons. Proceedings of the Royal Society Biological Sciences Series B. 278:3123-3128.

Titus RC. 1998. Short-range and long-range songs: Use of two acoustically distinct song classes by Dark-eyed Juncos. Auk. 115:386-393.

Ward AJW, Mehner T. 2010. Multimodal mixed messages: the use of multiple cues allows greater accuracy in social recognition and predator detection decisions in the mosquitofish, Gambusia holbrooki. Behavioral Ecology. 21:1315-1320.

Whittaker DJ, Richmond KM, Miller AK, Kiley , Bergeon Burns C, Atwell JW, Ketterson ED. 2011. Intraspecific preen oil odor preferences in dark-eyed juncos (Junco hyemalis). Behavioral Ecology. 22: 12561263. 